\title{
A New Estimator to Minimize the Error Due to Breathing in the Measurement of Respiratory Impedance
}

\author{
DANIEL NAVAJAS, RAMÓN FARRÉ, MAR ROTGER, AND RENÉ PESLIN
}

\begin{abstract}
This paper presents a new respiratory impedance estimator to minimize the error due to breathing. Its practical reliability was evaluated in a simulation using realistic signals. These signals were generated by superposing pressure and flow records obtained in two conditions: 1) when applying forced oscillation to a resistance-inertance-elastance (RIE) mechanical model; 2) when healthy subjects breathed through the unexcited forced oscillation generator. Impedances computed (4-32 Hz) from the simulated signals with the new estimator resulted in a mean value which was scarcely biased by the added breathing (errors less than 1 percent in the mean $R, I$, and $E$ ) and had a small variability (coefficients of variation of $R, I$, and $E$ of $1.3,3.5$, and 9.6 percent, respectively). Our results suggest that the proposed estimator reduces the error in measurement of respiratory impedance without appreciable extracomputational cost.
\end{abstract}

\section{INTRODUCTION}

$\mathrm{T}$ HE MECHANICAL input impedance measured by forced excitation characterizes the dynamic behavior of the respiratory system. The technique is based on submitting the respiratory system to a small amplitude and wide-band air perturbation while the subject breathes spontaneously. Given the fact that the subject breathes against the measuring device during forced excitation, a pressure is generated at the mouth due to the nonzero mechanical impedance of this device. Thus, two mechanical generators (i.e., the forced excitation device and the respiratory system) act simultaneously [4]. Hence, at frequencies where the activity of both generators is significant, the forced excitation signals are contaminated by the noises associated with the spontaneous breathing of the subject. In this situation where the input and the output of the system are corrupted by noises that are mutually correlated, impedance estimates calculated from the recorded signals are biased if the conventional cross-spectrum input-output estimators are used [1], [3], [4]. To reduce the bias error, Daroczy and Hantos [3] proposed a different alternative by using the driving voltage of the excitation device as a third signal to compute respiratory impedance. This approach is not generally used in prac-

Manuscript received February 8, 1988; revised July 25, 1988. This work was supported by the Comisión Asesora para la Investigacion Cientifica y Técnica (CAICYT 3132/83) and by Acción Integrada Hispano-Francesa $(18 / 208)$

D. Navajas, R. Farré, and M. Rotger are with the Lab. Biofisica i Bioenginyeria, Facultat de Medicina, Universitat de Barcelona, Barcelona, Spain.

R. Peslin is with Unité 14 INSERM de Physiopathologie Respiratoire, Vandoeuvre-Les-Nancy, France.

IEEE Log Number 8823684 tice since three instead of two signals must be processed. Moreover, the method is based on the assumption that the conversion of the driving voltage into the excitation pressure in the forced excitation device is linear [3], [5].

In this paper, a new respiratory impedance estimator to eliminate the bias error due to the correlation between pressure and flow breathing is proposed. It is based on using the available knowledge concerning the relation of the pressure and flow noises from the impedance of the measuring device. No hypothesis concerning the voltagepressure conversion is required. Moreover, this impedance estimator only requires the spectral estimation of the two usual recorded signals: pressure and flow. Thus, the computational time is not increased.

\section{THEORY}

Input respiratory impedance is estimated by forced excitation from pressure and flow recorded at the mouth. Fig. 1 shows a block diagram, in the frequency domain, of the signals involved. In this figure and the following, all signals are represented by their Fourier transforms. The dependence on frequency has been omitted to simplify the notation. $\dot{V}_{e}$ and $P_{e}$ are the excitation flow and pressure signals. $Z_{r s}$ is the input impedance of the respiratory system defined as follows:

$$
Z_{r s}=\frac{P_{e}}{\dot{V}_{e}} .
$$

$\dot{V}_{b}$ is the breathing flow and $P_{b}$ is the corresponding pressure generated. $P$ and $\dot{V}$ are the signals recorded by the transducers. $P_{b}$ and $\dot{V}_{b}$ act as noise corrupting the excitation signals:

$$
\begin{aligned}
& \dot{V}=\dot{V}_{e}+\dot{V}_{b} \\
& P=P_{e}+P_{b} .
\end{aligned}
$$

The pressure and flow breathing noises are related by means of the input impedance of the measuring device $Z_{e}$ :

$$
P_{b}=-Z_{e} \cdot \dot{V}_{b} .
$$

The negative sign in (4) is due to the opposite placement of the measuring device and the respiratory system in relation to the direction of flow. Substituting for $P_{e}$ and $P_{b}$ from (1) and (4) in (3) yields

$$
P=Z_{r s} \cdot \dot{V}_{e}-Z_{e} \cdot \dot{V}_{b} .
$$




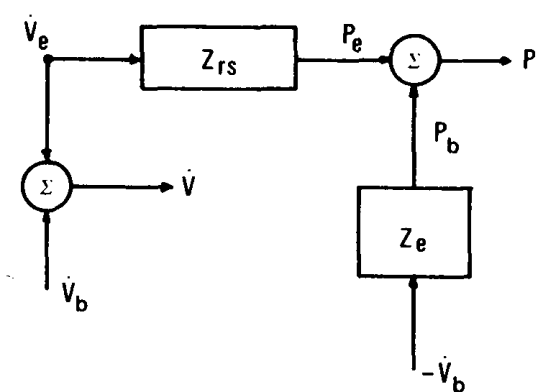

Fig. 1. Block diagram of excitation signals and noises.

Since $\dot{V}_{e}$ and $P_{e}$ are not directly measurable, $Z_{r s}$ is usually estimated from recorded $\dot{V}$ and $P$. The two estimators [6], [7] that are most widely used to calculate $Z_{r s}$ are

$$
\begin{aligned}
& Z_{1}=\frac{P \cdot \dot{V}^{*}}{\dot{V} \cdot \dot{V}^{*}}=\frac{G_{p v}}{G_{v v}} \\
& Z_{2}=\frac{P \cdot P^{*}}{\dot{V} \cdot P^{*}}=\frac{G_{p p}}{G_{v p}}
\end{aligned}
$$

where the superindex $*$ indicates a complex conjugate and $G_{x y}$ is defined as $X \cdot Y^{*}$. Applying (2) and (5) and making the generally accepted assumption [4], [5] that the forced excitation and the breathing signals are uncorrelated, it follows that

$$
\begin{aligned}
& Z_{1}=\frac{Z_{r s} \cdot G_{v e v e}-Z_{e} \cdot G_{v b v b}}{G_{v b v b} \cdot G_{v e v e}} \\
& Z_{2}=\frac{Z_{r s} \cdot Z_{r s}^{*} \cdot G_{v e v e}+Z_{e} \cdot Z_{e}^{*} \cdot G_{v b v b}}{Z_{r s}^{*} \cdot G_{v e v e}-Z_{e}^{*} \cdot G_{v b v b}} .
\end{aligned}
$$

Two major potential sources of error in $Z_{1}$ and $Z_{2}$ estimates arise. The first is a bias error due to the correlation between pressure and flow breathing noises [(4)]. Given that in conventional impedance measurements $Z_{e} \neq 0$ and $G_{v b v b} \neq 0$, the two estimators result in biased and different impedance estimates [(8) and (9)]. Moreover, as $Z_{1}$ and $Z_{2}$ depend on the value of $G_{v b v b}$, these biases are different for each subject and, thus cannot be corrected easily. If an ideal measuring device with $Z_{e}=0$ was available, $Z_{2}$ would be unbiased $\left(Z_{2}=Z_{r s}\right)$ whereas $Z_{1}$ would still remain biased $\left[Z_{1}=Z_{r s} \cdot G_{v e v e} /\left(G_{v b v b}+G_{v e v e}\right)\right]$. The second source is the bias and random statistical sampling errors inherent in the finite length of the pressure and flow records available in practice. Hence, only estimates of the true value of auto- and cross-spectra can be used to compute $Z_{1}$ and $Z_{2}$.

When respiratory impedance is estimated by means of $Z_{1}$ or $Z_{2}$, one proceeds as if no information relating $\dot{V}_{b}$ and $P_{b}$ was available. Although these breathing noises cannot be measured separately, we know the transfer function $Z_{e}$ of the system connecting them. This physical information is included in (5). Deducing $V_{e}$ from (2) and (5) gives

$$
\dot{V}_{e}=\frac{Z_{e} \cdot \dot{V}+P}{Z_{r s}+Z_{e}} .
$$

From (1), applying (2) and (3), and assuming that excitation and breathing signals are uncorrelated $\left(P_{b} \cdot \dot{V}_{e}^{*}=\right.$ $0 ; \dot{V}_{b} \cdot \dot{V}_{e}^{*}=0$ ), it follows that

$$
Z_{r s}=\frac{P_{e}}{\dot{V}_{e}}=\frac{P_{e} \cdot \dot{V}_{e}^{*}}{\dot{V}_{e} \cdot \dot{V}_{e}^{*}}=\frac{P \cdot \dot{V}_{e}^{*}-P_{b} \cdot \dot{V}_{e}^{*}}{\dot{V} \cdot \dot{V}_{e}^{*}-\dot{V}_{b} \cdot \dot{V}_{e}^{*}}=\frac{P \cdot \dot{V}_{e}^{*}}{\dot{V} \cdot \dot{V}_{e}^{*}} .
$$

Substituting for $\dot{V}_{e}$ from (10) yields

$$
Z_{r s}=\frac{Z_{e}^{*} \cdot P \cdot \dot{V}^{*}+P \cdot P^{*}}{Z_{e}^{*} \cdot \dot{V} \cdot \dot{V}^{*}+\dot{V} \cdot P^{*}}
$$

Hence, we define a new estimator $Z$ as follows:

$$
Z=\frac{Z_{e}^{*} \cdot G_{p v}+G_{p p}}{Z_{e}^{*} \cdot G_{v v}+G_{v p}}
$$

To compute $Z$ only one of the two cross-spectra has to be calculated in practice since $G_{v p}=G_{p v}^{*}$.

From (13), $Z=Z_{r s}$ provided that the true values for auto- and cross-spectra were used to compute $Z$. Consequently, the main source of bias error due to the correlation between pressure and flow breathing noises has been eliminated on estimator $Z$. Furthermore, only auto- and cross-spectra of the recorded pressure and flow signals are required to compute $Z$. Nevertheless, given that only records of finite length are available in practice to compte spectra, the potential source of bias and random statistical sampling errors remain. In order to evaluate the magnitude of these errors and the performance of $Z$ in practical impedance measurements, we carried out a simulation study using realistic excitation and breathing signals.

\section{Material and Methods \\ Simulation}

We used a forced-excitation device similar to those described in the literature [3], [4]. It was based on a 12 in loudspeaker attached to a 81 chamber. A bias tube connected to the chamber allowed the spontaneous breathing of the subject. Air in the device was renewed with a suction pump. The forced random noise excitation signal was generated by a microcomputer (IBM-PC/AT) and subsequently analogically low-pass filtered $(0-32 \mathrm{~Hz})$. Flow was recorded with a mesh-wire screen pneumotachograph and a differential pressure transducer (MP-45, Validyne, U.S.). Pressure was recorded with a similar transducer. Signals recorded by the transducers were analogically lowpass filtered $(0-32 \mathrm{~Hz})$ to prevent aliasing and digitized at $128 \mathrm{~Hz}$. The mechanical impedance of the excitation device $Z_{e}$ was measured by connecting a second loudspeaker to its outlet. The $Z_{e}$ modulus was $1.01 \mathrm{hPa} \cdot \mathrm{I}^{-1}$ - $s$ at $4 \mathrm{~Hz}$ and decreased slightly with frequency down to $1.00 \mathrm{hPa} \cdot 1^{-1} \cdot \mathrm{s}$ at $32 \mathrm{~Hz}$. This value of $Z_{e}$ is similar to others previously reported [4], [7]. 
We constructed a resistance-inertance-elastance (RIE) mechanical model by arranging in series a mesh-wire screen resistance, a rigid cylindrical tube and a rigid wall bottle. The impedance of this model was similar to that of a healthy subject (Table I). Forced excitation ( $\pm 1 \mathrm{hPa}$ ) was applied to the model. We obtained 16 different records (32 s each) of noise-free flow $\dot{V}_{e}$ and pressure $P_{e}$ excitation signals. Breathing noises were obtained from 16 healthy young volunteers ( 5 male, 11 female). Their spirometric indexes were within the normal range. For each subject, we recorded 32 s of flow $\dot{V}_{b}$ and pressure $P_{b}$ signals while the subject was spontaneously breathing through the measuring device, as in a real impedance test, but without applying any excitation signal to the loudspeaker. By adding one different $\dot{V}_{e}$ signal to each $\dot{V}_{b}$ noise, and similarly proceeding with the paired pressures, we synthesized 16 pairs of noisy pressure $P$ and flow $\dot{V}$ records. In this way, we generated an ensemble of 16 pressure and flow samples which simulated recordings of 16 subjects with different breathing patterns but each one with a respiratory impedance equal to that of the model. A section of a representative pair of flow and pressure records is shown in Fig. 2.

Each of the 32 s long $P$ and $\dot{V}$ synthesized records were digitally high-pass filtered (Butterworth, 3 poles, $3 \mathrm{~dB}$ at $2 \mathrm{~Hz}$ ) and divided into 63 blocks of $1 \mathrm{~s}$ (with 50 percent overlapping). These blocks were multiplied by a Hanning window and fast Fourier transforms (FFT) were computed. Averaging over the 63 blocks, an ensemble of 16 sample estimates of auto- and cross-spectra were computed [1]. These estimates were used to calculate $Z_{1}, Z_{2}$ and $Z[(6),(7)$, and (13)] and the corresponding coherence function $\gamma^{2}[1]$.

To evaluate the total bias and random error of each estimator, the mean value and standard deviation over the 16 values of $Z, Z_{1}$, and $Z_{2}$ were calculated. Finally, each obtained impedance curve was fitted to that of a RIE mathematical model by means of a standard least square algorithm [8]. Mean values and standard deviation of resistance, inertance, and elastance estimates were computed.

\section{RESULTS}

For each estimator, Fig. 3 shows the mean value and the standard deviation of the real and imaginary parts of the computed impedances of the 16 samples. In this figure, the model impedance, in the following named $Z_{r s}$, is also shown for reference. $Z_{r s}$ was calculated from the average of the auto- and cross-spectra of the 16 noise-free excitation records. With the proposed estimator $Z$ [Fig. 3(a)], the mean impedance clearly approached $Z_{r s}$. Fig. 3(b) and (c) correspond to $Z_{1}$ and $Z_{2}$ for the same simulated noisy records. In accordance with (8) and (9) for low frequencies where breathing noise contamination $\left(G_{v b v b}\right)$ was greater, the differences between the mean impedances and $Z_{r s}$ were clearly greater than in Fig. 3(a). The real part of $Z_{r s}$ was underestimated and its imaginary part was overestimated by $Z_{1}$ whereas the contrary behav.
TABLE 1

Mean and Standard Deviation of the Parameters

\begin{tabular}{|c|c|c|c|c|}
\hline & $Z_{r s}$ & $Z$ & $Z_{1}$ & $Z_{2}$ \\
\hline $\mathrm{R}$ & 2.32 & $\begin{array}{c}2.32 \pm 0.03 \\
(1.3 \\
\text { percent })\end{array}$ & $\begin{array}{c}2.16 \pm 0.12 \\
(5.5 \\
\text { percent })\end{array}$ & $\begin{array}{c}2.37 \pm 0.04 \\
(1.7 \\
\text { percent })\end{array}$ \\
\hline I & 1.14 & $\begin{array}{c}1.15 \pm 0.04 \\
(3.5 \\
\text { percent })\end{array}$ & $\begin{array}{c}1.07 \pm 0.05 \\
(4.7 \\
\text { percent })\end{array}$ & $\begin{array}{c}1.27 \pm 0.19 \\
(15.0 \\
\text { percent })\end{array}$ \\
\hline $\mathrm{E}$ & 53.0 & $\begin{array}{c}53.2 \pm 5.1 \\
(9.6 \\
\text { percent })\end{array}$ & $\begin{array}{c}44.3 \pm 5.9 \\
(13.3 \\
\text { percent })\end{array}$ & $\begin{array}{c}68.5 \pm 27.9 \\
(40.7 \\
\text { percent })\end{array}$ \\
\hline
\end{tabular}

Coefficients of variation in brackets. $R, I$, and $E$ in hPa $\cdot 1^{-1} \cdot \mathrm{s}, 10^{-2}$. $\mathrm{hPa} \cdot \mathrm{l}^{-1} \cdot \mathrm{s}^{2}$, and $\mathrm{hPa} \cdot 1^{-1}$, respectiv ly.
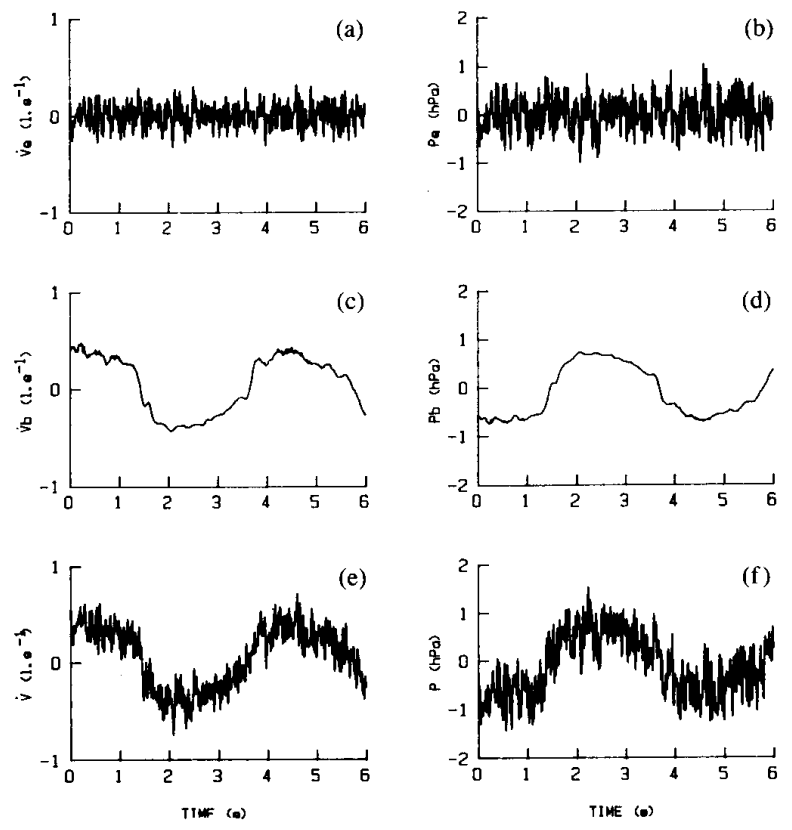

Fig. 2. Fragments of representative pairs of flow and pressure records used in the simulation. (a) and (b) flow $\left(\dot{V}_{e}\right)$ and pressure $\left(P_{e}\right)$ corresponding to the forced excitation. (c) and (d) flow $\left(\dot{V}_{b}\right)$ and pressure $\left(P_{b}\right)$ noises due to breathing. (e) and (f) noisy flow $(V)$ and pressure $(P)$ data used to compute impedance.

ior was found for $Z_{2}$. The differences between the mean values of the population and those of $Z_{r s}$ were greater for $Z_{1}$ than for $Z_{2}$ in the real part and greater for $Z_{2}$ than for $Z_{1}$ in the imaginary part. The standard deviations increased considerably in relation to those of $Z$ [Fig. 3(a)], especially in the case of $Z_{2}$.

The mean of the coherence function corresponding to the impedance estimates in Fig. 3 are depicted in Fig. 4. Up to around $12 \mathrm{~Hz}$ mean coherence increased with frequency. This was a consequence of the high-level of breathing noise contamination at the lowest frequencies. We obtained a mean value of $G_{v b v b} / G_{v e v e}$ of 0.41 at $4 \mathrm{~Hz}$ where coherence was around 0.5 . By contrast, at $12 \mathrm{~Hz}$ the mean value of $G_{v b v b} / G_{v e v e}$ was 0.02 where mean coherence was 0.96 . 


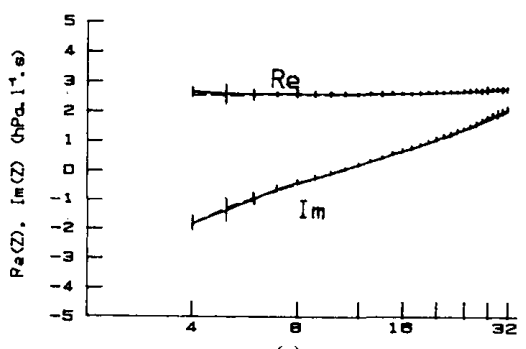

(a)

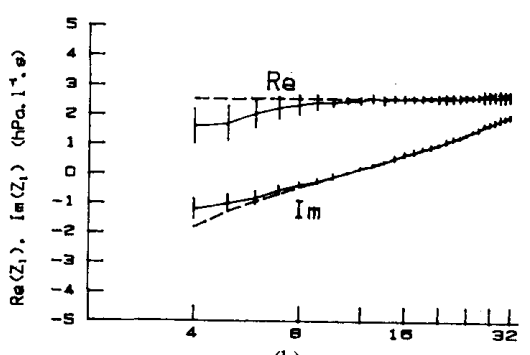

(b)

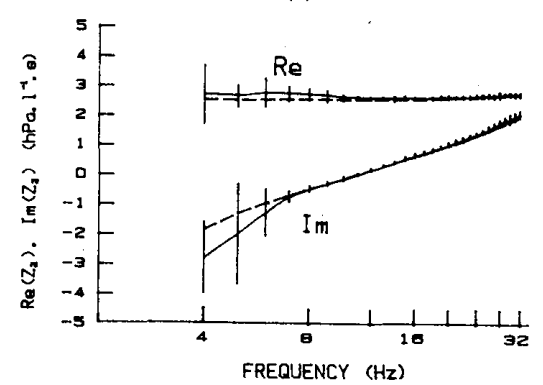

(c)

Fig. 3. Mean values and standard deviations of the real (Re) and imaginary $(\mathrm{Im})$ parts of estimated impedances. $Z_{r s}$ in dashed lines. (a) $Z$ estimator, (b) $Z_{1}$ estimator, (c) $Z_{2}$ estimator.

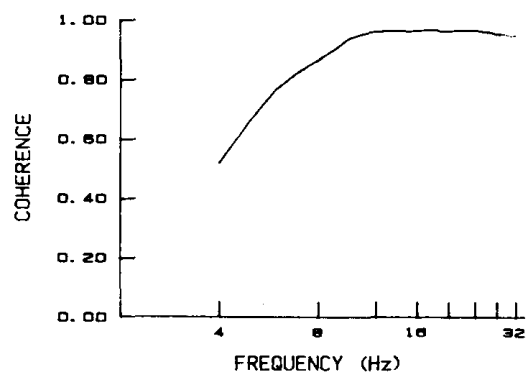

Fig. 4. Mean value of the coherence function between pressure and flow.

Parameter values in Table I quantifies the results shown in Fig. 3. This table shows the $R, I$, and $E$ values fitted from $Z_{r s}$. It also shows the mean and standard deviation of the parameters fitted from the impedance estimates of $Z, Z_{1}$, and $Z_{2}$. For the $Z$ estimator, the mean $R, I$, and $E$ were close to those of $Z_{r s}$ (differences of less than 1 percent) with coefficients of variation smaller than 2,4 , and 10 percent, respectively. By contrast, for the other estimators the mean $R, I$, and $E$ parameters differed from true values $\left(6.9,6.1\right.$, and 16.4 percent for $Z_{1}$ and $2.2,11.4$, and 29.2 percent, for $Z_{2}$, respectively) and the coefficients of variation of the parameters clearly increased with respect to those of $Z$.

\section{Discussion}

The estimator $Z$ proposed in this work was specifically designed to be applied to measurements of input respiratory impedance. It was defined from the equations linking the characteristics of the respiratory and measuring sytems $\left(Z_{r s}\right.$ and $\left.Z_{e}\right)$ with the excitation and breathing noise signals. In the theoretical approach followed to define $Z$, no special hypothesis has been made concerning the spectral characteristics of the forced excitation signal. Therefore, the proposed estimator can be used for any kind of excitation: random, pseudorandom, or sinusoidal. The hypothesis of uncorrelation between excitation and breathing is generally accepted [4], [5]. Furthermore, it was indirectly verified by Franken et al. [5] when the excitation signal was pseudorandom $(2,4,6, \cdots, 32 \mathrm{~Hz})$. As the mechanical impedance of the measuring device $Z_{e}$ is involved in the definition of $Z$ [(13)], it is assumed that $Z_{e}$ is constant when the device is submitted to the spontaneous breathing flow. This requirement, which is also implicitly assumed in the approach of Daroczy and Hantos [3], may be easily met by an adequate design of the excitation device. In contrast with the indirect estimation proposed by Daróczy and Hantos [3], no hypothesis concerning the linearity of the transformation of loudspeaker driving voltage to chamber pressure was required to define $Z$.

The performed simulation gave estimates of the expected value and variance when the impedance of a model was calculated from realistic noisy signals with a duration similar to those of usual impedance measurements. The simulation was carried out with a simple RIE stationary model since it is widely used to interpret respiratory impedance data of healthy subjects [5], [6]. Moreover, the impedance of this model is easily parametrized in terms of $R, I$, and $E$ by means of a linear procedure [8]. Nevertheless, as the theoretical unbiasness of $Z$ does not depend on the particular model used in a further analysis of the measured respiratory impedance, $Z$ may be applied when more complex models are used to fit experimental data. For instance, in the case of multicompartmental models, including partitioning of airways, alveolar gas, and chest wall. The results obtained showed that the estimator $Z$ was scarcely affected by the statistical sampling errors arising from the use of auto- and cross-spectral estimates obtained from short records of signals. As was expected from its definition, $Z$ showed a mean value which was scarcely biased even for mean coherences as low as 0.5 . By contrast, $Z_{1}$ and $Z_{2}$ resulted in values that were clearly biased, according to (8) and (9), and to the results reported by Daroczy and Hantos [3]. Moreover, the standard deviations of the estimated impedances (and also of the fitted parameters) were clearly smaller for $Z$ than for the other two estimators. This was also expected since $Z$ 
was defined to be unaffected by the breathing noises whereas $Z_{1}$ and $Z_{2}$ were not. The standard deviations of these two later estimators were due to the errors associated with the spectral estimates and to the variability of $G_{v b v b}$. For the population of healthy subjects studied in this work, we found that the coefficients of variation of $G_{v b v b}$ were around 100 percent at all frequencies. Furthermore, the improvement in impedance estimation observed in Fig. 3 and in Table I was achieved with the same cost of spectral estimation (FFT) since $Z$ only requires processing of the $P$ and $\dot{V}$ records.

The signal-to-noise ratio depends on the energy of the spontaneous breathing flow of each subject and on the energy of the forced excitation signal which cannot be arbitrarily increased. At low frequencies where the breathing energy is great, only low signal-to-noise ratios may be achieved during forced excitation, especially in patients [2]. Thus, if a biased estimator is used, impedance measurements may become useless even when prolonging the measuring time since increasing the signal length would only reduce random errors but not bias [4], [5]. In contrast, the calculation of $Z$ from signal records that are sufficiently long to reduce random errors would provide a reliable impedance estimation for low values of the signal-to-noise ratio. The prolongation of the measuring time is not difficult to carry out in clinical practice since patient fatigue may be avoided by allowing pauses between forced excitation recordings. Our simulation study suggests that the use of the $Z$ estimator could extend the frequency range of reliable measurements to lower frequencies, thus providing more physiological and clinical information from respiratory impedance tests.

\section{ACKNOWLEDGMENT}

The authors wish to thank Dr. Z. Hantos and Dr. B. Daróczy for their valuable criticism and A. Martin for his technical assistance.

\section{REFERENCES}

[1] J. S. Bendat and A. G. Piersol, Random Data: Analysis and Measurement Procedures. New York: Wiley, 1971.

[2] J. Clement, F. J. Landser, and K. P. Van de Woestijne, "Total resistance and reactance in patients with respiratory complaints with and without airways obstruction," Chest, vol. 83, pp. 215-220, 1983.

[3] B. Daróczy and Z. Hantos, "An improved forced oscillatory estimation of respiratory impedance," Int. J. Biomed. Comput., vol. 13, pp. 221-235, 1982.

[4] E. Delavault, G. Saumon, and R. Georges, "Characterization and validation of forced input method for respiratory impedance measurement," Resp. Physiol., vol. 40, pp. 119-136, 1980.

[5] H. Franken, J. Clement, and K. P. Van de Woestijne. "Systematic and random errors in the determination of respiratory impedance by means of the forced oscillation technique: A theoretical study," IEEE Trans. Biomed. Eng., vol. BME-30, pp. 642-651, 1983.

[6] E. D. Michaelson, E. D. Grassman, and W. R. Peters, "Pulmonary mechanics by spectral analysis of forced random noise," J. Clin. Invest., vol. 56, pp. 1210-1230, 1975.
[7] T. K. Miller, III, and R. L. Pimmel, "Standard errors on respiratory mechanical parameters obtained by forced random excitation," IEEE Trans. Biomed. Eng., vol. BME-30, pp. 826-832, 1983.

[8] M. Tsai and R. L. Pimmel, "Computation of respiratory resistance, compliance, and inertance from forced oscillatory impedance data," IEEE Trans. Biomed. Eng., vol. BME-26, pp. 492-493, 1979.

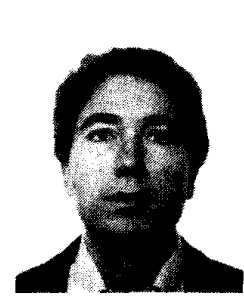

Daniel Navajas was born in Barcelona, Spain, in 1950. He received the Ph.D. degree in physics in 1980 from the University of Barcelona, Barcelona, Spain.

He is Professor of Physiology at the Faculty of Medicine University of Barcelona. Since 1980 he has been engaged in respiratory biophysics and bioengineering. His current research interest includes respiratory mechanics, modeling, digital signal processing, transducers, and instrumentation.

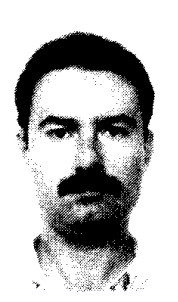

Ramón Farré was born in Barcelona, Spain, in 1957. He received the Ph.D. degree in physics in 1988 from the University of Barcelona, Barcelona, Spain.

He is an Associate Professor at the Faculty of Medicine, University of Barcelona. Since 1982 he has been working in the field of respiratory mechanics, particularly in the modeling of the respiratory system by means of its mechanical impedance, and in the design and characterization of pressure and flow transducers.

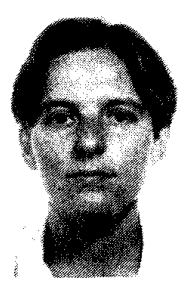

Mar Rotger was born in Badalona, Spain. She received the $\mathrm{Ph}$. D. degree in physics in 1986 from the University of Barcelona, Barcelona, Spain.

She is a Professor of Physiology at the Faculty of Medicine, University of Barcelona. Her principal research interest is digital signal processing and modeling of the respiratory system.

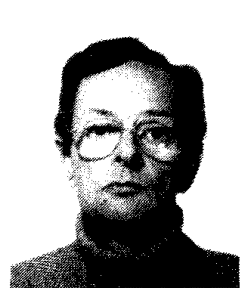

René Peslin was born in France in 1937. He received the M.D. degree in 1964 from the University of Nancy, Nancy, France.

He has been a Full-Time Researcher at the Institut National de la Santé et de la Recherche Médicale (INSERM). Presently, he is the Director of INSERM research Unit nr 14, which is devoted to experimental and clinical work in the field of respiratory clinical physiology. His own research field is respiratory mechanics, particularly the development of noninvasive methods to explore lung and airway properties. 